\title{
THE IMMERSION OF MANIFOLDS
}

\author{
BY S. GITLER AND M. MAHOWALD ${ }^{1}$
}

Communicated by N. E. Steenrod, April 21, 1967

1. M. Hirsch [3] has shown that the immersion problem for manifolds is just a cross section problem for the stable normal bundle. Our object here is to find conditions under which sections of the tangent bundle will imply sections in the normal bundle (and conversely). First we need some notation.

Given an integer $t$, let $j(t)$ be the maximum integer such that the $2^{t}$-fold Whitney sum of the Hopf bundle over $R P^{j(t)-1}$ is trivial. If $\xi$ is a stable bundle, let $\operatorname{gd}(\xi)$ denote the geometric dimension of $\xi$.

THEOREM A. Let $M^{m}$ be an m-dimensional manifold, $m \leqq 2^{t}-1$, whose stable tangent bundle $\tau_{0}$ is trivial over the $(j(t)-1)$-skeleton. If $m-j(t)+1$ is odd or if $H^{q}\left(M ; Z_{p}\right)=0$ for all $p \neq 2$ and $q \neq 0, m$, then $\operatorname{gd}\left(\tau_{0}\right) \leqq m-j(t)+1$ implies $\operatorname{gd}\left(-\tau_{0}\right) \leqq m-j(t)+1$.

To illustrate the strength of Theorem A we offer

TheOREM B. Let $m=2^{t}$, then $R P^{m-1}$ immerses in $R^{2 m-j(t)+1}$ but not in $R^{2 m-j(t)}$.

The negative result in Theorem $B$ is due to James [4]. Milgram in [8] has obtained linear immersions of $R P^{m}$ which agree with those of Theorem B only if $m=15$ and 31 .

2. Outline of the proofs. Let $X$ be a space, then by $X[k]$ we denote the $k$ th-Eilenberg subcomplex of the space $X$, i.e., $X[k]$ is $(k-1)$ connected and there is a map $f: X[k] \rightarrow X$ such that $f_{*}: \pi_{q}(X[k])$ $\cong \pi_{q}(X)$ for $q \geqq k$. Let $B O_{n}$ and $B O$ denote, respectively, the classifying spaces of $n$-plane bundles and stable bundles. The natural map $B O_{n} \rightarrow B O$ induces maps $p: B O_{n}[k] \rightarrow B O[k]$ for all $k$.

The key step in the proof of Theorem $B$ is

Theorem C. For each $m<2^{t}$ there exists an $H$-space $E$, an $H$-map $\phi: E \rightarrow B O[j(t)]$ and a fiber map $\psi: B O_{n(t, m)}[j(t)] \rightarrow E$ such that

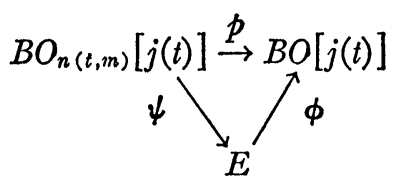

${ }^{1}$ The second author is an A.P. Sloan Fellow and was partially supported by the U.S. Army Research Office (Durham). 
is commutative, where $n(t, m)=m-j(t)+1$. Let $F$ be the fiber of $\psi$. If $n(t, m)$ is odd, then $F$ is m-connected. If $n(t, m)$ is even, then $F$ is $(n(t, m)-1)$-connected and $\pi_{k}(F)$, for $n(t, m) \leqq k \leqq m$ is either zero or a finite group of odd order.

We will now outline the proof of Theorem C. We will assume $t$ and $m$ are fixed integers.

As in [2] or [5], we can construct a fiber space $\phi_{0}: X \rightarrow B O$ as a composite of principal fiber spaces

$$
X=X_{s} \rightarrow X_{s-1} \rightarrow \cdots \rightarrow X_{0}=B O
$$

where $X_{k} \rightarrow X_{k-1}$ has fiber a product of Eilenberg-Mac Lane spaces of type $(Z, q)$ or $\left(Z_{2}, q\right)$. There is a fiber map $\psi_{0}: B O_{n} \rightarrow X$ such that

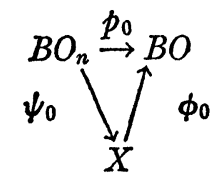

is commutative. If $F$ is the fiber of $\psi_{0}$, then $F$ satisfies the conditions of Theorem $\mathrm{C}$ and we have,

Proposition 2.3. Let $t>4$, then the diagram of Theorem $\mathrm{C}$ is induced by diagram (2.2) under the natural map $B O[j(t)] \rightarrow B O$.

Because of (2.3), it suffices to prove that $E$ is an $H$-space and that $\phi$ is an $H$-map.

Now the fiber space $E \rightarrow B O[j(t)]$ is a composite of principal fibrations,

$$
E=E_{s} \rightarrow E_{s-1} \rightarrow \cdots \rightarrow E_{0}=B O[j(t)]
$$

induced by (2.1). Let $F_{k}$ be the fiber of $E_{k} \rightarrow E_{k-1}$. Then $E_{k} \rightarrow E_{k-1}$ is classified by a map $f_{k}: E_{k-1} \rightarrow B F_{k}$ where $B F_{k}$ is the classifying space of $F_{k}$. Assume that $E_{k-1}$ is an $H$-space and that $\phi_{k-1}: E_{k-1} \rightarrow E_{0}$ is an $H$-map. Then $E_{k}$ will be an $H$-space if the $k$-invariants, i.e. the images of the fundamental classes of $B F_{k}$ are primitive in $H^{*}\left(E_{k-1}\right)$. We have a principal fiber space

$$
K\left(J_{t}, j(t-1)-1\right) \rightarrow B O[j(t)] \rightarrow B O[j(t-1)]
$$

where $J_{t}=Z$ or $Z_{2}$, accordingly with [1]. Let

$$
\bar{E}=\bar{E}_{s} \rightarrow \cdots \rightarrow \bar{E}_{0}=K\left(J_{t}, j(t-1)-1\right)
$$

be the induced fibrations over $\bar{E}_{0}$ of (2.4). 
Stong in [9], has determined the mod 2-cohomology of $B O[n]$. Using this result we prove,

Proposition 2.6. For $k=0,1, \cdots, s$, the natural map $\bar{E}_{k} \rightarrow E_{k}$ induces a monomorphism $H^{*}\left(E_{k}\right) \rightarrow H^{*}\left(\bar{E}_{k}\right)$ in dimensions $\leqq 2^{t}-1$.

Again inductively, if we assume that $E_{k-1}$ is an $H$-space, then $\bar{E}_{k-1}$ is an $H$-space and $\bar{E}_{k-1} \rightarrow E_{k-1}$ is an $H$-map. Therefore from (2.6) follows

Proposition 2.7. If the k-invariants of $\bar{E}_{k} \rightarrow \bar{E}_{k-1}$ are primitive, then $E_{k}$ and $\bar{E}_{k}$ are $H$-spaces and $E_{k} \rightarrow \bar{E}_{k}$ is an $H$-map.

In fact, we show that $\bar{E}_{k}$ is an infinite loop space. Again inductively, if $\bar{E}_{k-1}$ is an infinite loop space and $\Omega^{-r} \bar{E}_{k-1}$ denotes a space such that $\Omega^{r}\left(\Omega^{-r} \bar{E}_{k-1}\right)=\bar{E}_{k-1}$, it suffices to show that the $k$-invariants of $\bar{E}_{k} \rightarrow \bar{E}_{k-1}$ are in the image of

$$
\Sigma^{r} \bar{E}_{k-1} \stackrel{g}{\rightarrow} \Omega^{-r} \bar{E}_{k-1} \rightarrow \Omega^{-r} B F_{k}
$$

where $g$ is homotopic to the adjoint of the identity map $\bar{E}_{k-1} \rightarrow \bar{E}_{k-1}$.

In order to achieve this, we pass to Thom complexes. First observe that for $r \geqq 2^{t}, \bar{E}_{0}$ is the fiber of $B O_{r}[j(t)] \rightarrow B O_{r}[j(t-1)]$. Assume from now on, that $r \geqq 2^{t}$. Let

$$
E^{\prime}=E_{s}^{\prime} \rightarrow E_{s-1}^{\prime} \rightarrow \cdots \rightarrow E_{0}^{\prime}=B O_{r}[j(t)]
$$

be the tower induced over $E_{0}^{\prime}$ from (2.4). Then the tower (2.5) is induced from (2.9) by the mapping $\bar{E}_{0} \rightarrow E_{0}^{\prime}$. Now let $\eta_{r}$ be the canonical bundle over $E_{0}^{\prime}$ and consider the induced bundles over the spaces $E_{k}^{\prime}$ and $\bar{E}_{k}$. Let $M E_{k}^{\prime}$ and $M \bar{E}_{k}$ denote the corresponding Thom complexes. We have a sequence of maps

$$
M E^{\prime}=M E_{s}^{\prime} \rightarrow M E_{s-1}^{\prime} \rightarrow \cdots \rightarrow M E_{0}^{\prime} .
$$

Lemma 2.11. In dimensions less than or equal to $r+m$, the map $M E_{k}^{\prime} \rightarrow M E_{k-1}^{\prime}$ looks like a principal fiber map, with fiber a product of Eilenberg-Mac Lane complexes and $k$-invariants $\left\{U \cup k_{i}\right\}$, where the $k_{i}$ are the $k$-invariants for $E_{k}^{\prime} \rightarrow E_{k-1}^{\prime}$.

Let $Y_{q}$ be the universal example of an integral cohomology class $y$ of dimension $q$ such that

(1) all primary cohomology operations vanish on $y$,

(2) all operations that raise dimension by less than $j(t)$ vanish on $y$.

Then we need the following 
THEOREM 2.12. There exists a tower of fiber spaces

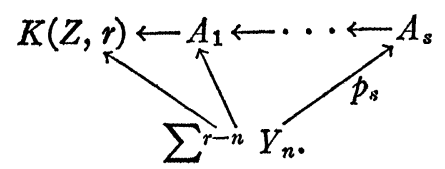

The fiber $G_{k}$ at each stage is a product of Eilenberg-MacLane spaces. If $k>1$, then $G_{k}=\Omega^{-r} F_{k} \times S_{k}$ where $F_{k}$ is the $(k-1)$-stage of an Adams resolution over $Z_{2}$ of $V_{n}$ through dimension $m-1$ and $S_{k}$ is the $k$-stage of an Adams resolution over $Z_{2}$ of $S^{r}$ through dimension $\Gamma+j(t)-1$. Also $G_{1}=\prod_{i=1}^{t-1} K\left(Z_{2}, r+2^{i}-1\right) \times \Omega^{-r} F_{1}$. In addition, the fiber of $p_{s}$ is $(m-1)$ connected.

The proof of (2.12) follows closely that of [6, Theorem A].

Let $f_{k}$ be the composite $A_{k} \rightarrow B G_{k+1} \rightarrow \Omega^{-r-1} F_{k+1}$ where the first map classifies $A_{k+1} \rightarrow A_{k}$ and the second is the obvious projection.

TheOREM 2.13. There is a mapping $\lambda_{k-1}: M E_{k-1}^{\prime} \rightarrow A_{k-1}$ such that $\lambda_{k-1}^{*} f_{k-1}^{*}\left(\gamma_{i}\right)=U \cup k_{i}$, where $\gamma_{i}$ ranges over the fundamental classes of $\Omega^{-r-1} F_{k}$ and the $k_{i}$ over the $k$-invariants of $E_{k}^{\prime} \rightarrow E_{k-1}^{\prime}$.

Now we are ready to indicate how Theorem $C$ follows. Observe that $M \bar{E}_{k}=\Sigma^{r}\left(\bar{E}_{k} \cup_{p t}\right)$, since the induced bundle over $\bar{E}_{k}$ is trivial, and let $i_{k}: \Sigma r \bar{E}_{k} \rightarrow M E_{k}^{\prime}$ be the composite $\Sigma^{r} \bar{E}_{k} \rightarrow M \bar{E}_{k} \rightarrow M E_{k}^{\prime}$.

Suppose that we have proved $\bar{E}_{k}$ has the structure of an infinite loop space, then the following lemma implies $\bar{E}_{k+1}$ has the structure of an infinite loop space:

LEMma 2.14. There is a commutative diagram, up to homotopy,

$$
\begin{array}{cc}
\Sigma^{r} \bar{E}_{k} \stackrel{\rho_{k}}{\rightarrow} \Omega^{-r} \bar{E}_{k} \\
\downarrow i_{k} \stackrel{\downarrow j}{ } \quad j_{k} \\
M E_{k}^{\prime} \stackrel{\lambda_{k}}{\rightarrow} \quad A_{k} \stackrel{f_{k}}{\rightarrow} B F_{k+1}
\end{array}
$$

where $\lambda_{k}$ is given in (2.13). $\rho_{k}$ is the adjoint of the identity map and $f_{k} j_{k}$ classifies the bundle $\Omega^{-r} \bar{E}_{k+1} \rightarrow \Omega^{-r} \bar{E}_{k}$.

The proof of Theorem $\mathrm{C}$ is then completed.

Theorem A follows by establishing a $\bmod p$ version of (2.13) and using the fact that the top class of the Thom complex of the normal bundle to a manifold immersed in euclidean space is stably spherical.

Finally, Theorem B follows from Theorem A by using the existence of tangent vector fields for real projective spaces due to HurwitzRadon. 


\section{REFERENCES}

1. R. Bott, The stable homotopy of the classical groups, Ann. of Math. (2) 70 (1959), 313-337.

2. S. Gitler and M. Mahowald, Geometric dimension of stable vector bundles, (2) Bol. Soc. Mat. Mexicana 11 (1966).

3. M. Hirsch, Immersion of manifolds, Trans. Amer. Math. Soc. 93 (1959), 242276.

4. I. M. James, On the immersion of real projective spaces, Bull. Amer. Math. Soc. 69 (1963), 231-238.

5. M. Mahowald, On obstruction theory in orientable fiber bundles, Trans. Amer. Math. Soc. 110 (1964), 315-349.

6. - On the metastable homotopy of $S^{n}$, Mem. Amer. Math. Soc. No. 72 (1967).

7. M. Mahowald and F. Peterson, Secondary cohomology operations in the Thom complex, Topology 2 (1964), 367-377. 482.

8. R. S. Milgram, Immersing projective spaces, Ann. of Math. (2) 85 (1967), 473-

9. R. Stong, Determination of $H^{*}\left(B O(k, \cdots, \infty), Z_{2}\right)$ and $H^{*}(B U(k, \cdots, \infty)$, $\left.Z_{2}\right)$, Trans. Amer. Math. Soc. 107 (1963), 526-544.

Centro de Investigacion del IPN and

NORTHWESTERN UNIVERSITY 